\title{
Tidal Dwarf Galaxy Candidates in Hickson Compact Groups of Galaxies
}

\author{
Philippe Amram ${ }^{1}$, Claudia Mendes de Oliveira ${ }^{2}$, Henri Plana ${ }^{3}$, Chantal \\ Balkowski ${ }^{4}$. \\ 1 Observatoire de Marseille, France \\ 2 Instituto de Astronomia, São Paulo, Brazil \\ 3 Universidade de Santa Cruz, Brazil \\ 4 Observatoire de Paris-Meudon, France
}

\begin{abstract}
The detailed study of the internal kinematics of tidal dwarf galaxy (TDG) candidates detected in optical and/or HI observations is most important to address the question about their origins. We are conducting a program to measure the kinematics of TDG candidates in compact groups using a Fabry-Perot instrument which allows us to study the $2-\mathrm{D}$ velocity maps of the objects with high spatial and spectral resolutions. Our main goal is to select TDG candidates which have kinematics independent of the possible parent galaxies. In this paper, we describe our results for Stephan's quintet (SQ) and HCG 31. We find that the dynamical masses of the TDG candidates in SQ range from $10^{8}$ up to $10^{10}$ $M_{\odot}$. Two objects to the $\mathrm{E}$ and $\mathrm{S}$ of HCG $31 \mathrm{~A}+\mathrm{C}$ present counterrotating patterns with amplitudes of 30 and $40 \mathrm{~km} \mathrm{~s}^{-1}$. Region $\mathrm{F}$, which was previously thought to be the best example of TDG presents no rotation.
\end{abstract}

Compact groups (CGs) have been identified as sites of galaxy formation, with the identification of a number of tidal-dwarf galaxy candidates associated with the tidal tails of their member galaxies. In fact, Hunsberger et al. (1996) found that about one fifth of the Hickson CGs have candidate tidal dwarf galaxies. Nevertheless, that study is based on $\mathrm{R}$ images only and the candidates needed to be confirmed kinematically. Our observations were, then, carried out with a Fabry-Perot instrument mounted on the ESO 3.6m telescope. The pixel size on the sky was 0.91 arcsec; the field of view was 170 " $\times 170$ " and the velocity sampling $16 \mathrm{~km} \mathrm{~s}^{-1}$. The data reduction procedure has been extensively described (Amram et al. 1998 and references therein).

In our study of SQ (Plana et al. 1999; Mendes de Oliveira et al. 2001), we identified seven TDG candidates (see right panel of Fig. 1) for which we derived velocity fields and rotation curves. These have the right magnitudes $\left(\mathrm{M}_{B}=-16\right.$ to -11.7$)$, sizes $(4-7 \mathrm{kpc})$, colours $(\mathrm{B}-\mathrm{R}=0.3-0.8)$ and gas velocity gradients $(\sim 50 \mathrm{~km} / \mathrm{s})$ to be TDGs. Two of the candidates are associated with HI clouds, one of which is, in addition, associated with a $\mathrm{CO}$ cloud. All seven regions have low continuum fluxes and high $\mathrm{H} \alpha$ luminosities $(1-60) \times 10^{-14} \mathrm{erg}$ $\mathrm{s}^{-1} \mathrm{~cm}^{-2}$. Their masses range from $2 \times 10^{8}$ to $10^{10} \mathrm{M}_{\odot}$ and the median value for their inferred $M / L$ is $7(M / L)$. At least two of them may survive possible "fallbacks" or disruption by the parent galaxies. SQ is known to be a nursery of young objects. Besides the TDG candidates mentioned above, young clusters 
and more recently intergalactic HII regions (IHII) have been identified in this group (Mendes de Oliveira et al. 2003, see Fig 1, left panel).

Our main findings on HCG 31 are (Amram et al. 2004, in preparation): (1) the group clearly contains an ongoing merger that involves members $\mathrm{A}$ and $\mathrm{C}$, given that a double kinematic component is present throughout the main body of $\mathrm{A}+\mathrm{C},(2)$ two regions to the $\mathrm{E}$ and $\mathrm{S}$ of $\mathrm{A}+\mathrm{C}$, present rotation patterns with amplitudes of 30 and $40 \mathrm{~km} \mathrm{~s}^{-1}$ and, moreover, they counterrotate with respect to $\mathrm{A}+\mathrm{C},(3)$ region $\mathrm{F}$, which was previously thought to be the best example of a tidal dwarf galaxy in HCG 31, is indeed a tidal debris following the same kinematic pattern of the parent galaxy and presenting no rotation. HCG 31 presents an undergoing merger in its center $(\mathrm{A}+\mathrm{C})$; moreover, several evidences for interaction with the other galaxy components of the group $(B, Q$ and $G)$ indicate that the complex $\mathrm{A}+\mathrm{C}$ is probably accreting the surrounding galaxies.

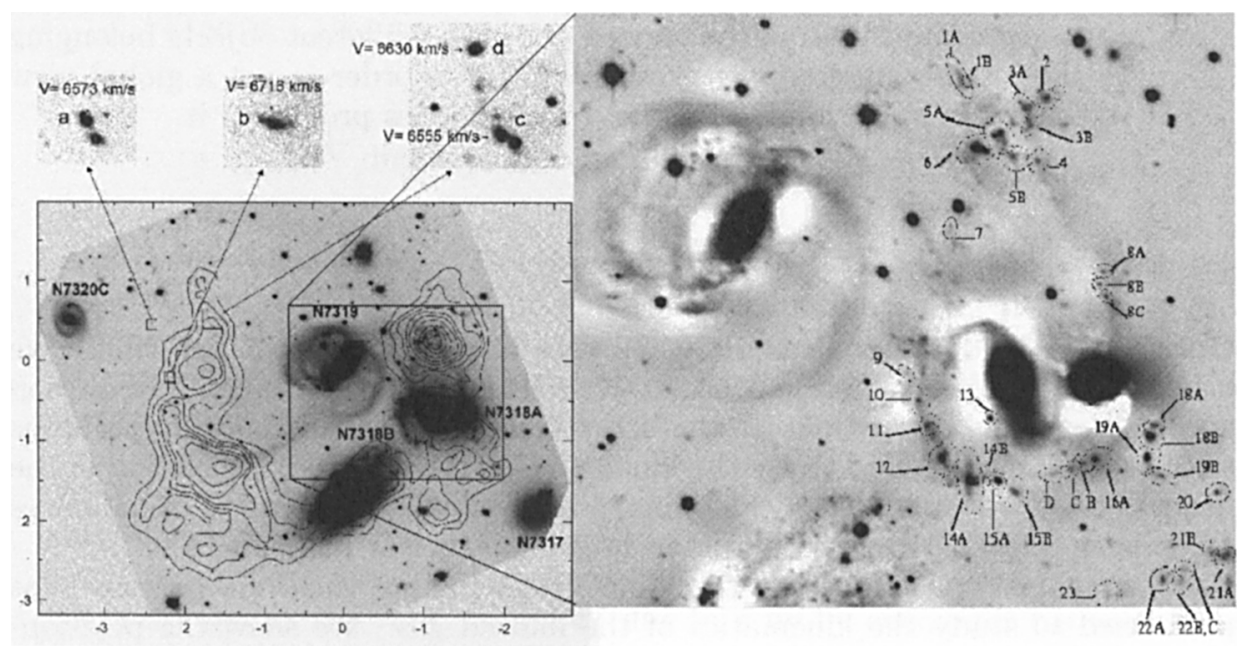

Figure 1. Stephan's Quintet (HCG 92). LEFT: HI contours (from Williams et al. 2002) are overplotted on an R-band image. Intergalactic HII regions are zoomed in on the top images (Mendes de Oliveira et al, 2003). RIGHT: Enlargement of the central area of the group (R-image after sky substraction). The seven TDG candidates are 2-5, 6, 8, 20, 21,22 and 23.

\section{References}

Amram P. et al. 1998 A\&A, 330, 881

Hunsberger S.D., Charlton J.C, Zaritsky D., 1996, ApJ, 462, 50

Mendes de Oliveira C., Plana H., Amram P. et al, 2001, AJ, 121, 252

Mendes de Oliveira C. et al., 2003, AJ, in press.

Plana H., Mendes de Oliveira C., Amram P. et al 1999, AJ, 516, L69

Williams, B.A., Yun, M.S., Verdes-Montenegro, L., 2002, AJ, 123, 241 